\title{
A Framework for Risk-Energy Aware Service-Level Agreement Provisioning (RESP) for Computing the Quickest Path
}

\author{
Ashutosh Sharma $(\mathbb{D})$ and Rajiv Kumar \\ Department of Electronics and Communication, Jaypee University of IT, Solan, India \\ Correspondence should be addressed to Ashutosh Sharma; sharmaashutosh1326@gmail.com
}

Received 31 August 2018; Revised 13 March 2019; Accepted 14 March 2019; Published 6 May 2019

Academic Editor: Juraj Machaj

Copyright (c) 2019 Ashutosh Sharma and Rajiv Kumar. This is an open access article distributed under the Creative Commons Attribution License, which permits unrestricted use, distribution, and reproduction in any medium, provided the original work is properly cited.

\begin{abstract}
This paper involved the computation of quickest paths with the help of service-level agreement (SLA) and energy constraints. The consideration of these constraints helps to propose a novel system model which computes the path to strengthen the continuity and criticality of the data transmission. The proposed risk-energy aware SLA provisioning (RESP) system model incorporates the research gaps of the existing risk assessment models in the literature without any increase in time complexity. The formulation of RESP shows the usefulness in the critical applications. The results show that the computation of risk-provisioned path gives the SLA satisfied paths and has a great impact of variations in the data traffic. The performance of the proposed algorithm has been indicated in terms of different performance parameters such as mean $s-t$ paths, average hop counts, and average energy efficiency. Simulation result implies that as data traffic increases, the number of the risk-provisioned $s-t$ paths decreases whereas corresponding average hop counts and average energy efficiency increases.
\end{abstract}

\section{Introduction}

Due to the advancements in the technology of the computer networks, the applications associated with network have been provided by the service providers. These applications have a great impact on the life of human beings [1]. Therefore, a dependable network has to be requested for the network applications $[2,3]$. In this regard, the concerns of environmentalists have been raised for the consumption of minimum energy for the design of dependable network [4]. Recently, the concept of dependability and energy has been considered together to address performability engineering [5]. The performance of a computer network relies mainly on the reliability, bandwidth, delay, and energy of the links which participate during data transmission. In this paper, an attempt has been performed to consider these parameters for computing the quickest path problem between two specific ends, i.e., source and destination.

With evolution of network services, quickest path problem (QPP) was proposed by the authors in [6] for the data transmission. A polynomial algorithm was proposed for the data transmission to find a solution for QPP in the generalized computer network. Later, this problem got attention from several researchers, and authors in [7] modified the problem by considering the idea of bicriterion for the range of different data. This problem extends for the singlepair QPP to all-pair QPP as shown by the authors in [8]. Several extensions have been made for the QPP as the k-QPP was introduced in [9-12]. For general network, it is very important to design a routing protocol for high availability so that critical services may get completed. To design a routing protocol for high availability, the parameters like energy, delay, and bandwidth are the major factors which play an important role $[13,14]$. This QPP model was also extended toward the building evacuation problem by Hamacher and Tjandra [15] who propose a building evacuation problem. The QPP model was also extended toward the continuity of critical applications where it has been considered as an important figure of merit [16].

The network is associated with certain uncertainties which may lead to service failure, and to deal with these failures, reliability theory has been used in [17] and the reliable QPP model has been proposed. This model was later extended for different models in [12, 18-20]. Also, authors in 
[19] extend the reliable QPP by considering a threshold value of reliability for quality-of-service (QoS) routing. Later, the reliable model was extended for risk analysis by considering service-level agreement (SLA) [21-25].

Recently, QoS routing has been provided by considering the amount of energy used for the data transmission services by considering parameters like reliability, risk, and availability. [26, 27]. The reliable QPP problem further extended with energy constraint for the continuity of data transmission [28]. The discussion made in the above paragraphs leads us to work for continuity of critical data transmission services by provisioning SLA and energy. Recently, risk-energy problem in data transmission services has been discussed for minimization in the risk and energy consumption [29].

1.1. Research Significance. Here, in this paper, the authors have made an attempt to provision the risk-free path computation model with consideration of SLA and energy constraints. The main contributions of this paper are presented as follows: (i) A novel system model has been presented with the risk-provisioned mechanism which is the upgraded version of the existing REQPP risk assessment system model in the existing literature. (ii) The contribution can also be seen with the reference to SLA violation factor. The proposed system model gives the full SLA satisfaction as compared to the existing REQPP system model.

The rest of the paper is organized into sections. Section 2 presents the preliminaries to develop the strong base for the development of the system model. A novel system model is proposed in Section 3. To implement the proposed system model, an algorithm and its complexity analysis are presented in Section 4. Section 5 presents the results and discussion of the proposed problem. In Section 6, conclusion is presented.

\section{Preliminaries}

To explain the QPP model, let us consider the directed loopless graph $G=(N, E)$, where $N$ and $E$ are the set of number of nodes and set of number of links $(u, v)$, respectively. This network is associated with several resources such as link delays $d(u, v)>0$ and link capacities $c(u, v)>0$. Assume a data $\sigma$ transmission has been considered between two consecutive links with a connection-oriented approach having no buffer, i.e., seamless flow of data. To aid the seamless flow, capacity of a $s-t$ path has to be least and is defined as the data sent over a link per unit time.

Let us assume that $\sigma$ amount of data is being transmitted between two specific ends, namely, source $s$ and destination $(t)$. While data are being transferred between these two specific ends, a $s-t$ path is formed with the sequence of the number of links without any loop to manage the resources efficiently.

To transmit $\sigma$ unit's data over a $s-t$ path, the delay experienced at a link $(u, v)$ can be expressed as $T_{\sigma}(u, v)=d(u, v)+(\sigma / c(u, v))$. Let a path $P=\left(s=u_{1}, u_{2}\right.$, $\left.u_{3}, \ldots, u_{k}=t\right)$ where $u_{i} \in N, i=1, \ldots, k$ and $\left(u_{i}, u_{i+1}\right) \in E$, $i=1, \ldots, k-1$ be the loop-free $s-t$ path. Therefore, the minimum transmission delay experienced to transmit $\sigma$ unit's data along a $s-t$ path is

$$
T_{\sigma}(P)=d(P)+\left[\frac{\sigma}{c(P)}\right]
$$

where first term in the right hand side is known as the delay of path $d(P)$ calculated as $d(P)=\sum_{i=1}^{i=k-1} d\left(u_{i}, u_{i+1}\right)$ and the numerator of the second term is known as the minimum capacity for the $s-t$ path $c(P)=\min _{i=1}^{k-1} c\left(u_{i}, u_{i+1}\right)$.

Hence, the minimum transmission time is given as

$$
T_{\sigma}(P)=\sum_{i=1}^{i=k-1} d\left(u_{i}, u_{i+1}\right)+\left\lceil\frac{\sigma}{\min _{i=1}^{k-1} c\left(u_{i}, u_{i+1}\right)}\right\rceil .
$$

The quickest path problem (QPP) is formulated as $\min T_{\sigma}(P)$,

s.t. $P$ is an $s-t$ path in network $G(N, E)$.

In the beginning, when QPP was conceptualized $[6-11,14,15,30,31]$, no special care was maintained for the energy consumption during data transmission. Nowadays, without the consideration of energy [26], any one cannot afford transmission $[27,28,32]$. In addition to this, the bar on usage of energy resources put a constraint for data transmission [33-39]. To consider the concept energy consumption, the topic of green computing was explored in $[40,41]$ for data transmission.

To deal with energy, various authors tried to incorporate them in QPP. Let us assume that each link is allied with energy rate $\omega(u, v)>0$ to transmit $\sigma$ unit data from node $u$ along with the link $(u, v)$. In networks, each node is endowed with limited amount of power $P_{u}$ which is available at nodes for data transmission. The amount of energy required at the node is calculated as $\omega(u, v)(\sigma / c(u, v))$.

To compete with successful data transmission, the following has to be proved:

$$
P_{u}-\omega(u, v) \frac{\sigma}{c(u, v)} \geq 0 .
$$

For data transmission without any disruption, find minimum link capacity used for transmission of $\sigma$ unit data between nodes $u$ and $v$ having energy rate $\omega(u, v)$. The minimum capacity of link is given as follows:

$$
c_{\min E}(u, v)=\min _{i=1, \ldots, r}\left\{c_{i} ; P_{u}-\omega(u, v) \frac{\sigma}{c_{i}} \geq 0\right\} .
$$

In the given network, each link follows the following equation for the path capacity path $c(P)$ :

$$
c(u, v) \geq c_{j} \geq c_{\min }(u, v), \quad \text { where } j=1, \ldots, r .
$$

The residual energy of path $P_{u}(\sigma, P)$ is

$$
P_{u}(\sigma, P)= \begin{cases}P_{u}-\omega\left(u_{i}, u_{i+1}\right) \frac{\sigma}{c(P)}, \\ & \text { if } u=u_{i}, i=1, \ldots, k-1, \\ P_{u}, & \text { otherwise. }\end{cases}
$$


Hence, constrained QPP has been formulated by using above equations as

$$
\begin{array}{ll}
\min _{P} & T_{\sigma}(P), \\
\text { s.t. } & P_{u}(\sigma, P) \geq 0, u \in P, P \text { is an } s-t \text { path in network } G .
\end{array}
$$

The above-discussed model has been proposed by several authors $[28,42,43]$ to incorporate energy in QPP.

The availability of sufficient amount of energy is not only the factor for the continuity in data transmission. The link reliabilities are also helpful for the continuity of data transmission $[44,45]$.

Recently, the authors in [42] tried to adopt the above constraints in their proposed model, but they have incorporated these parameters for the assessment of risk unless provisioning for the risk. In the proposed framework, the authors have made an attempt to deal with the provisioning of risk.

\section{Proposed System Model}

3.1. Assumptions. The proposed system model for the RESP required some assumptions for better understanding and they are given as follows:

(1) There are no parallel and duplicate links in the network graph [46]

(2) Capacities of links are generated randomly with the uniform distribution and are statistically independent [47]

(3) Bifurcation of data is not allowed, and also, flow of conservation is followed by data transmission [47]

Let us take the performance reliability which constitutes the link reliability $R_{\mathrm{l}}(u, v)$ and performance factor of a link $R_{\mathrm{D}}(u, v)$. The reliability of a link is related to the failure in the connectivity (i.e., hard failure), and the performance factor of a link is related to the performance parameters such as delay and capacity (i.e., soft failure) [42].

Let us assume that link reliability is ideal, i.e., $R_{\mathrm{l}}(u, v)=1$ and performance of data transmission depends mainly on the performance factor. The requested service-level agreement (SLA) is drawn for the critical data transmission. The requested SLA is defined as $\left(a, t_{\mathrm{p}}\right)$ where $a$ is the requested SLA availability and $t_{\mathrm{p}}$ is the penalty period. By using these SLA parameters, the allowed time $T_{\text {A.D. }}$ has been computed as $[21,42]$

$$
T_{\text {A.D. }}=(1-a) \times t_{p}
$$

The link performance factor constitutes the service-level agreements (SLAs) and link delays; therefore, along a path, the performance factor is calculated as

$$
R_{\mathrm{D}}(P)=\prod_{i=1}^{k-1} e^{\left(\left(-T_{\sigma}\left(u_{i}, u_{i+1}\right)\right) / T_{A . \mathrm{D} .}\right)} .
$$

Equation (10) is known as the performance reliability due to delay $\left(R_{\mathrm{D}}\right)$, and by expanding equation (10), performance reliability is calculated as

$$
\begin{aligned}
& R_{\mathrm{D}}(P)=e^{\left(\left(-\sum_{i=1}^{k-1} T_{\sigma}\left(u_{i}, u_{i+1}\right)\right) / T_{\text {A.D. }}\right)}, \\
& R_{\mathrm{D}}(P)=e^{\left(-T_{\sigma}(P) / T_{\text {A.D. }}\right)} .
\end{aligned}
$$

In equation (11), if minimum transmission delay $T_{\sigma}(P)$ is very large as compared to $T_{\text {A.D. }}$, then the value of performance delay $R_{\mathrm{D}}(P)$ is equal to zero and if minimum transmission delay $T_{\sigma}(P)$ is small as compared to $T_{\text {A.D. }}$, then the value of performance delay $R_{\mathrm{D}}(P)$ is equal to 1 . The above study shows that the minimum transmission delay has a great impact on the performance of the data transmission.

Here, in this paper, the authors have tried to incorporate the research gaps of the existing risk assessment REQPP model proposed in [42]. In the REQPP model, the authors performed only the assessment of risk and not the provisioning. In the present paper, following this research gap, the authors have tried to incorporate risk provisioning by satisfying the SLAs and proposed the novel system model for risk provisioning.

A path $P$ is formed either by combination of several links or a single link. Therefore, it is more realistic to satisfy the SLA piecewise or between two consecutive links other than satisfying the SLA after completion of data transmission service among the path. The SLAs are considered for mission-critical applications; therefore, each node is endowed with the requested amount of reliability $\left(R_{u}\right)$. To deal with SLA constraint, the following expression has to follow:

$$
e^{\left((-d(u, v)+(\sigma / c(u, v))) / T_{\text {A.D. }}\right)} \geq R_{u}, \quad \forall(u, v) \in E,
$$

where $R_{u}$ is the reliability at nodes and has to be maintained equal or more to this value.

The minimum SLA aware link capacity is shown as follows:

$$
c_{\operatorname{min~SLA}}(u, v)=\min _{i=1, \ldots, r}\left\{e^{\left(-\left[d(u, v)+\left(\sigma / c_{i}\right)\right] / T_{\text {A.D. }}\right)}-R_{u} \geq 0\right\} .
$$

To incorporate both parameters (energy and SLA) for sorting, the minimum link capacity has been given as follows:

$$
\begin{aligned}
c_{\min }(u, v)= & \min \left\{\left[c_{\min E}(u, v) \geq c_{\mathrm{a}} \geq c(u, v)\right]\right. \\
& \left.\cap\left[c_{\min S L A}(u, v) \geq c_{\mathrm{b}} \geq c(u, v)\right]\right\},
\end{aligned}
$$

where $c_{\mathrm{a}}$ is the capacity lying between the minimum energy cooperative capacity and link capacity. The capacity $c_{\mathrm{b}}$ lies between the minimum SLA cooperative capacity and link capacity Equation (14) provides the label of minimum link capacity to support the continuity and criticality in data transmission if $c_{\text {min SLA }}(u, v)$ and $c_{\min E}(u, v)>0$. A $s-t$ path $P$ is feasible if $c(P) \geq c_{\min }(u, v)$. The above equations sort the minimum capacity which incorporates both the critical and continuous data transmission by considering the AND rule.

The remaining value of performance reliability is termed as residual-requested performance reliability. $R_{u}(\sigma, P)$ 
along the path $P$ gives feasibility of path $P$, i.e., $R_{u}(\sigma, P)$ $\geq 0, \forall u \in P$ as

$$
R_{u}(\sigma, P)=\left\{\begin{array}{l}
-\ln \left(R_{u}\right)-\left\{-\ln \left[e^{\left(\left(-\left[d\left(u_{i}, u_{i+1}\right)+(\sigma / c(P))\right]\right) / T_{\text {A.D. }}\right)}\right]\right\}, \\
\text { if } u=u_{i}, \quad i=1,2, \ldots, k-1, \\
-\ln \left(R_{u}\right), \quad \text { otherwise. }
\end{array}\right.
$$

The SLA-energy awareness allows us to combine the characteristics of the both models (energy and SLA) and formulate the constraint model of the risk-energy aware SLA provisioning (RESP) given as follows:

$$
\begin{array}{ll}
\min _{P} & T_{\sigma}(P), \\
\text { s.t. } & R_{u}(\sigma, P) \geq 0, \quad u \in P, \\
& P_{u}(\sigma, P) \geq 0, \quad u \in P, \\
& P \text { is an } s-t \text { path in network } G .
\end{array}
$$

Here, problem has been used to transmit the data with continuity and criticality using shortest path problem (SPP). The energy and SLA have been used as a constraint for the SPP. The working of SPP lies on Dijkstra's algorithm with the link delay as a cost value in the different subnetworks [48]:

$$
\begin{array}{rl}
\mathrm{SPP}_{j}: \quad \min _{P} & d(P), \\
\text { s.t. } & P \text { is a } s-t \text { path in the network } G_{j} .
\end{array}
$$

By following this, Algorithm 1 has been proposed in Section 4 to perform the computations.

\section{Algorithm}

\subsection{Algorithm for Risk-Energy Aware SLA Provisioned (RESP) Route.}

\subsection{Complexity Analysis}

Theorem 1. The time complexity of the proposed RESP algorithm is $O(r(m+n(\log (n))))$.

Proof. The complexity of RESP is explained using the time complexity of $O(m+n(\log n))$ [49] which has been run for $r$ times.

\section{Results and Discussion}

5.1. Experimental Setup. The experiment for the performance evaluation of the proposed RESP algorithm has been performed over the personal computer having the configuration of Intel(R) Core ${ }^{\mathrm{TM}_{\mathrm{i}}}$-7400, CPU@ $3.00 \mathrm{GHz}, 8 \mathrm{~GB}$ RAM, and Windows 10 operating system. All simulations have been performed in MATLAB R2010a environment platform. The simulations show that the proposed RESP algorithm is solvable in polynomial time using Dijkstra's algorithm which used binary heap data structure. The illustrations of results have been explained with the help of standard network topologies as shown in Figure 1(a) and $1(\mathrm{~b})$.

The associated values of link parameters like delay and capacities are taken from the uniform distribution range [1, 1000]. As discussed in the previous sections, the amount of fixed powers at each node and amount of energy rate at each link is given by $P_{u}=3 \times 10^{7}$ and $10^{-4} c(u, v) d^{2}(u, v)$, respectively. The requested reliability at nodes is generated uniformly within the range $[0,1]$. For the risk provisioning, requested SLA is defined by considering the availability and the penalty time $\left(a, t_{\mathrm{p}}\right)$. The value of requested availability is taken as $a=0.92$, and the penalty time is considered as $\left(t_{\mathrm{p}}=40000 \mathrm{secs}\right)$. To visualize the performance of the proposed algorithm, three different amounts of data $\left(\sigma_{1}=100, \sigma_{2}=1000\right.$, and $\sigma_{3}=10000$ in $\left.\mathrm{Mb}\right)$ are taken. This variation in the amount of data to be transmitted shows the trend in the various SLAs and energy-provisioned shortest paths.

5.2. Performance Analysis. The quantitative performance analysis of the proposed algorithm has been shown in $\mathrm{Ta}$ bles 1 and 2 . The different simulations have been performed for different data sizes. The set of different number of $s-t$ paths has been commutated from the different subgraphs which are equal to distinct capacities present in the network. Further, from the set of $s-t$ paths, the shortest path has been computed. The performance of proposed RESP algorithm has been seen in other parameters also such as average hop counts, average capacity, and energy efficiency of riskprovisioned $s-t$ shortest path. The results for both standard topologies have been taken for the discussion to show the effectiveness of the RESP.

5.2.1. 24-Node US Network. The results for the standard topology as shown in Figure 1(a) show the mean number of $s-t$ shortest paths computed for the ten runs. Table 1 shows the results for risk-provisioned $s-t$ paths in second, third, and fourth columns for different data traffics $\sigma_{1}, \sigma_{2}$, and $\sigma_{3}$. The mean value of risk-provisioned $s-t$ paths shows that as data traffic is varied, the number of $s-t$ paths got reduced. This trend has been shown because for the large amount of data, the links in the network topology are not capable to provide the energy and SLA provisioning. Also, hop counts in fifth, sixth, and seventh columns of Table 1 shows very little variation with respect to different data traffics.

The trend in the adopted capacity of path is increased as the data traffic increases as shown in the eighth, ninth, and tenth columns of Table 1, respectively. This trend has been found because for the large amount of data, the capacity of link should be maintained maximum.

5.2.2. 14-Node NSF Network. The experiment conducted for the Figure 1(a) has been repeated for the Figure 1(b) also to see the performance of the proposed RESP algorithm. The results for the 14-node NSF topology are shown in Table 2. The results for this topology depict that the same trend of the performance has been followed. 
Input: $G(N, E), \sigma, \omega(u, v), c(u, v), d(u, v), a, R_{u}, P_{u}$ and $t_{p}$

Output: risk-energy aware SLA-provisioned (RESP) route

BEGIN \{

Initialization:

$j \longleftarrow 1$,

\section{Procedure:}

STEP 0: variable declaration

$G \longleftarrow$ directed network

$N \longleftarrow$ set of nodes

$E \longleftarrow$ set of links

$c(u, v) \longleftarrow$ capacity of the link $(u, v)$

$d(u, v) \longleftarrow$ delay of link the $(u, v)$

$\omega(u, v) \longleftarrow$ energy rate of link the $(u, v)$

$P_{u} \longleftarrow$ endowed energy at node $u$

$R_{u} \longleftarrow$ requested reliability at node $u$

$\sigma \longleftarrow$ data

$t_{\mathrm{p}} \longleftarrow$ penalty time

$a \longleftarrow$ availability

STEP 1: prune $r$ different capacities of links corresponds to critical-continuous service and label of minimum capacity:

(i) $c_{1}<c_{2}<c_{3}<\cdots<c_{r}$

(ii) $c_{\min }(u, v)$ with AND rule

STEP 2: solve $S P P_{j}$ w.r.t delay time $d(u, v)$ in $G_{j}$ with capacities $c_{j}$

For $j \longleftarrow 1: r$

Set $j \longleftarrow 1$

Solve $S P P_{j}$

If No $s-t$ path in $G_{j}$ with capacities $c_{j}$ go to STEP 3

else end

Let $P_{j}$ is an optimal solution for $S P P_{j}$ with capacity $c\left(P_{j}\right)=c_{j}$

end

STEP 3:

For $j \longleftarrow r$

go to STEP 4

else

set $j=j+1$ and go to STEP 2

end

STEP 4: find the solution

Find index $h \in(1,2, \ldots, r)$ of path array $P_{j}$ such that

$T_{\sigma}\left(P_{\mathrm{h}}\right)=\min _{j=1, \ldots, r} T_{\sigma}\left(P_{j}\right)$

$P_{\mathrm{h}}$ is an optimal solution of the RESP

\}END

Algorithm 1: RESP algorithm.

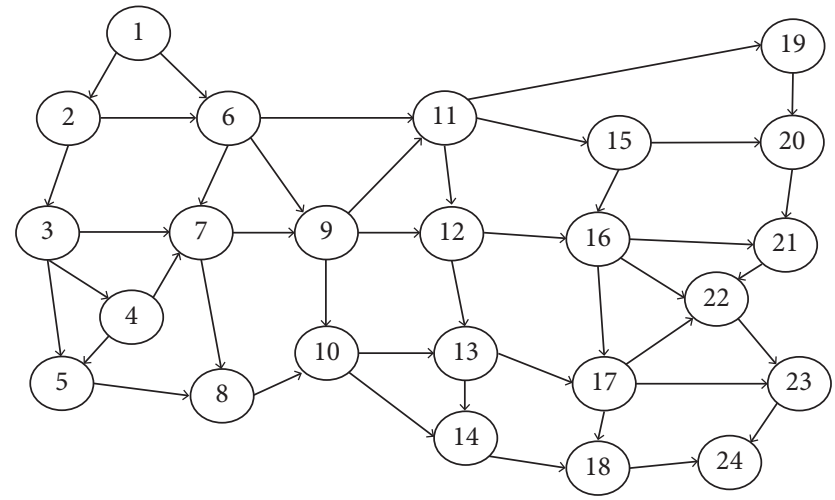

(a)

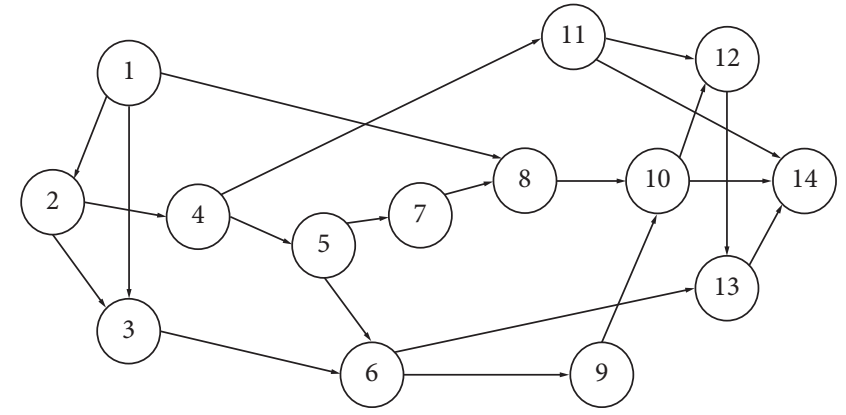

(b)

FIGURE 1: (a) 24-node US-directed mesh network with $s=1$ and $t=24$. (b) 14-node NSF-directed mesh network with $s=1$ and $t=14$. 
TABLE 1: Number of $s-t$ paths, hop counts, capacity, and energy efficiency of the shortest $s-t$ path.

\begin{tabular}{|c|c|c|c|c|c|c|c|c|c|c|c|c|}
\hline \multirow{3}{*}{ Sr. no. } & \multicolumn{12}{|c|}{ Traffic } \\
\hline & \multicolumn{3}{|c|}{$s-t$ paths } & \multicolumn{3}{|c|}{$\begin{array}{l}\text { Hop counts of } \\
\text { shortest } s-t \text { path }\end{array}$} & \multicolumn{3}{|c|}{$\begin{array}{c}\text { Capacity of shortest } \\
s-t \text { path }\end{array}$} & \multicolumn{3}{|c|}{$\begin{array}{l}\text { Energy efficiency of shortest } \\
\qquad s-t \text { path }\left(\times 10^{6}\right)\end{array}$} \\
\hline & $\sigma_{1}$ & $\sigma_{2}$ & $\sigma_{3}$ & $\sigma_{1}$ & $\sigma_{2}$ & $\sigma_{3}$ & $\sigma_{1}$ & $\sigma_{2}$ & $\sigma_{3}$ & $\sigma_{1}$ & $\sigma_{2}$ & $\sigma_{3}$ \\
\hline 1 & 13 & 10 & 4 & 7 & 8 & 8 & 12 & 20 & 12 & $1.0933 \mathrm{MJ}$ & 0.2727 & 0.1968 \\
\hline 2 & 8 & 9 & 6 & 8 & 10 & 8 & 7 & 25 & 29 & $0.2539 \mathrm{MJ}$ & 0.2846 & 0.2339 \\
\hline 3 & 9 & 10 & 11 & 7 & 6 & 6 & 25 & 38 & 69 & $0.3633 \mathrm{MJ}$ & 0.4422 & 0.3351 \\
\hline 4 & 5 & 8 & 5 & 7 & 7 & 6 & 12 & 16 & 29 & 0.1174 & 0.2571 & 0.4326 \\
\hline 5 & 5 & 12 & 10 & 7 & 8 & 7 & 7 & 11 & 48 & 0.1455 & 0.1021 & 0.3536 \\
\hline 6 & 10 & 9 & 4 & 7 & 7 & 6 & 12 & 38 & 27 & 0.2531 & 0.7795 & 0.5594 \\
\hline 7 & 11 & 7 & 6 & 7 & 7 & 7 & 3 & 12 & 19 & 0.00375 & 0.4081 & 0.1346 \\
\hline 8 & 13 & 8 & 10 & 7 & 7 & 7 & 2 & 33 & 45 & 0.0557 & 0.4670 & 0.5593 \\
\hline 9 & 7 & 6 & 10 & 7 & 7 & 7 & 29 & 15 & 35 & 0.5187 & 0.1670 & 0.8984 \\
\hline 10 & 9 & 9 & 8 & 7 & 8 & 8 & 4 & 49 & 30 & 0.0915 & 0.4998 & 0.6006 \\
\hline Mean & 9 & 8.8 & 7.4 & 7.1 & 7.5 & 7 & 11.3 & 25.7 & 34.3 & 0.289615 & 0.36801 & 0.43043 \\
\hline
\end{tabular}

TABLE 2: Number of $s-t$ paths, hop counts, capacity, and energy efficiency of the shortest $s-t$ path.

\begin{tabular}{|c|c|c|c|c|c|c|c|c|c|c|c|c|}
\hline \multirow{3}{*}{ Sr. no. } & \multicolumn{12}{|c|}{ Traffic } \\
\hline & \multicolumn{3}{|c|}{$s-t$ paths } & \multicolumn{3}{|c|}{$\begin{array}{l}\text { Hop counts of } \\
\text { shortest } s-t \text { path }\end{array}$} & \multicolumn{3}{|c|}{$\begin{array}{c}\text { Capacity of shortest } \\
s-t \text { path }\end{array}$} & \multicolumn{3}{|c|}{$\begin{array}{l}\text { Energy efficiency of shortest } \\
\qquad s-t \text { path }\left(\times 10^{6}\right)\end{array}$} \\
\hline & $\sigma_{1}$ & $\sigma_{2}$ & $\sigma_{3}$ & $\sigma_{1}$ & $\sigma_{2}$ & $\sigma_{3}$ & $\sigma_{1}$ & $\sigma_{2}$ & $\sigma_{3}$ & $\sigma_{1}$ & $\sigma_{2}$ & $\sigma_{3}$ \\
\hline 1 & 6 & 6 & 4 & 3 & 4 & 4 & 9 & 37 & 47 & 0.1264 & 0.9527 & 1.1781 \\
\hline 2 & 7 & 3 & 2 & 3 & 3 & 4 & 15 & 22 & 16 & 0.3360 & 2.6999 & 0.3110 \\
\hline 3 & 3 & 7 & 3 & 4 & 4 & 4 & 19 & 35 & 22 & 1.3800 & 2.1949 & 0.5721 \\
\hline 4 & 5 & 3 & 5 & 4 & 3 & 4 & 24 & 23 & 39 & 0.3952 & 0.2289 & 0.4877 \\
\hline 5 & 3 & 5 & 5 & 5 & 4 & 3 & 7 & 19 & 24 & 0.2209 & 0.6922 & 0.4690 \\
\hline 6 & 6 & 6 & 6 & 4 & 3 & 4 & 16 & 27 & 27 & 0.3795 & 0.3887 & 0.9240 \\
\hline 7 & 2 & 6 & 3 & 3 & 3 & 4 & 7 & 14 & 34 & 0.1651 & 0.7255 & 0.8131 \\
\hline 8 & 5 & 3 & 2 & 5 & 3 & 4 & 10 & 15 & 18 & 0.7991 & 0.2552 & 0.8775 \\
\hline 9 & 6 & 3 & 4 & 4 & 5 & 4 & 14 & 19 & 27 & 1.1382 & 0.9686 & 0.3725 \\
\hline 10 & 9 & 6 & 2 & 4 & 4 & 3 & 53 & 33 & 28 & 0.9906 & 0.2891 & 0.5150 \\
\hline Mean & 5.2 & 4.8 & 3.6 & 3.9 & 3.6 & 3.8 & 17.4 & 24.4 & 28.2 & 0.5931 & 0.93957 & 0.652 \\
\hline
\end{tabular}

The variation of data traffic has high impact on the energy efficiency (bits/sec/joule) of $s-t$ path in the given topology. The results for the energy efficiency have been shown in the eleventh to thirteenth columns. The value of energy efficiency increased as data traffic increases. This is because the energy constraints have to be qualified to be part of a subnetworks, so as data traffic increases, energy efficiency also increases.

The illustrative results show that the proposed RESP algorithm is outperforming over the REQPP algorithm [42] with reference to risk provisioning, resource wastage, and computation time.

\section{Conclusion and Future Work}

This paper has proposed the concept of risk-energy aware SLA provisioning (RESP) for the planning of critical routing where continuity of data transmission is given utmost priority. Continuity of transmission in this paper has been quantified in terms of performance of the link in a data transmission path considered as energy required for the link connectivity. The link delay has also been considered for the risk provisioning. The result shows an improvement in energy efficiency and capacity of path. The proposed RESP algorithm is able to compute the paths without any risk in terms of SLA. Present work is considering only energy of the link in path computation. In future work, the proposed algorithm can be extended with incorporating the security paradigms which enable the computation of paths in presence of any malicious activities.

\section{Data Availability}

No data have been used separately for the presentation of the work. Each and every detail of the results has been included already in the Results section.

\section{Conflicts of Interest}

The authors declare that they have no conflicts of interest.

\section{Acknowledgments}

The authors are thankful for the financial grant for this paper from the research project titled "Reliability Modeling and Optimized Planning of Risk-Based Resilient Networks" 
sponsored by Indo-Polish Program under grant DST/INT/ POL/P-04/2014.

\section{References}

[1] C. R. Kalmanek, S. Misra, and Y. R. Yang, Guide to Reliable Internet Services and Applications, Springer Science \& Business Media, Berlin, Germany, 2010.

[2] L. Norros, I. Norros, M. Liinasuo, and K. Seppänen, "Impact of human operators on communication network dependability," Cognition, Technology \& Work, vol. 15, no. 4, pp. 363-372, 2013.

[3] A. Arroyo and T. Grisham, Leading Extreme Projects: Strategy, Risk and Resilience in Practice, Taylor \& Francis, Abingdon, UK, 2017.

[4] P. E. Heegaard, B. E. Helvik, and V. B. Mendiratta, "Achieving dependability in software-defined networking-a perspective," in Proceedings of the 7th International Workshop on Reliable Networks Design and Modeling (RNDM), pp. 63-70, Munich, Germany, October 2015.

[5] K. B. Misra, Handbook of Performability Engineering, Springer Science \& Business Media, Berlin, Germany, 2008.

[6] Y. L. Chen and Y. H. Chin, "The quickest path problem," Computers \& Operations Research, vol. 17, no. 2, pp. 153-161, 1990.

[7] E. D. Q. V. Martins and J. L. E. Dos Santos, "An algorithm for the quickest path problem," Operations Research Letters, vol. 20, no. 4, pp. 195-198, 1997.

[8] J. B. Rosen, S.-Z. Sun, and G.-L. Xue, "Algorithms for the quickest path problem and the enumeration of quickest paths," Computers \& Operations Research, vol. 18, no. 6, pp. 579-584, 1991.

[9] M. M. B. Pascoal, M. E. V. Captivo, and J. C. N. Clímaco, “An algorithm for ranking quickest simple paths," Computers \& Operations Research, vol. 32, no. 3, pp. 509-520, 2005.

[10] M. M. B. Pascoal, M. E. V. Captivo, and J. C. N. Clímaco, "A comprehensive survey on the quickest path problem," Annals of Operations Research, vol. 147, no. 1, pp. 5-21, 2006.

[11] M. Pascoal, M. E. Captivo, and J. C. Clímaco, "Computational experiments with a lazy version of a $K$ quickest simple path ranking algorithm," TOP, vol. 15, no. 2, pp. 372-382, 2007.

[12] S. Ruzika and M. Thiemann, "Min-max quickest path problems," Networks, vol. 60, no. 4, pp. 253-258, 2012.

[13] J. Rak, Resilient Routing in Communication Networks, Springer, Berlin, Germany, 2015.

[14] D. Eppstein, "Finding the k shortest paths," SIAM Journal on Computing, vol. 28, no. 2, pp. 652-673, 1998.

[15] H. W. Hamacher and S. A. Tjandra, "Mathematical modelling of evacuation problems: a state of art," in Pedestrian and Evacuation Dynamics, Springer, Berlin, Germany, 2001.

[16] P. H. Pathak and R. Dutta, Designing for Network and Service Continuity in Wireless Mesh Networks, Springer Science \& Business Media, Berlin, Germany, 2012.

[17] G. Xue, "End-to-end data paths: quickest or most reliable?," IEEE Communications Letters, vol. 2, no. 6, pp. 156-158, 1998.

[18] S. Tragoudas, "The most reliable data-path transmission," IEEE Transactions on Reliability, vol. 50, no. 3, pp. 281-285, 2001.

[19] H. I. Calvete, L. del-Pozo, and J. A. Iranzo, "Algorithms for the quickest path problem and the reliable quickest path problem," Computational Management Science, vol. 9, no. 2, pp. 255-272, 2012.

[20] Y.-K. Lin, "Extend the quickest path problem to the system reliability evaluation for a stochastic-flow network,"
Computers \& Operations Research, vol. 30, no. 4, pp. 567-575, 2003.

[21] M. Xia, M. Tornatore, C. U. Martel, and B. Mukherjee, "Riskaware provisioning for optical WDM mesh networks," IEEE/ ACM Transactions on Networking, vol. 19, no. 3, pp. 921-931, 2011.

[22] K. Vajanapoom and D. Tipper, "Risk based incremental survivable network design," in Proceedings of the 6th International Workshop on Design and Reliable Communication Networks, pp. 1-9, La Rochelle, France, October 2007.

[23] K. Vajanapoom, Risk-Based Survivable Network Design, University of Pittsburgh, Pittsburgh, PA, USA, 2008.

[24] K. Vajanapoom, D. Tipper, and S. Akavipat, "Risk based resilient network design," Telecommunication Systems, vol. 52, no. 2, pp. 799-811, 2013.

[25] A. J. Gonzalez and B. E. Helvik, "SLA success probability assessment in networks with correlated failures," Computer Communications, vol. 36, no. 6, pp. 708-717, 2013.

[26] P. Chołda and P. Jaglarz, "Optimization/simulation-based risk mitigation in resilient green communication networks," Journal of Network and Computer Applications, vol. 59, pp. 134-157, 2016.

[27] P. Chołda and P. Jaglarz, "Energy-efficiency versus resilience: risk awareness view on dimensioning of optical networks with a sleep mode," Photonic Network Communications, vol. 30, no. 1, pp. 43-58, 2015.

[28] H. I. Calvete, L. del-Pozo, and J. A. Iranzo, "The energyconstrained quickest path problem," Optimization Letters, vol. 11, no. 7, pp. 1319-1339, 2017.

[29] Y. Wu, M. Tornatore, C. U. Martel, and B. Mukherjee, "Green and low-risk content placement in optical content delivery networks," in Proceedings of the IEEE International Conference on Communications (ICC), pp. 1-6, Kuala Lumpur, Malaysia, May 2016.

[30] S. Ruzika and M. Thiemann, "Reliable and restricted quickest path problems," in Network Optimization, pp. 309-314, Springer, Berlin, Germany, 2011.

[31] T. Gomes and J. Craveirinha, "Efficient calculation of the most reliable pair of link disjoint paths in telecommunication networks," European Journal of Operational Research, vol. 181, no. 3, pp. 1055-1064, 2007.

[32] J. Chabarek, J. Sommers, P. Barford, C. Estan, D. Tsiang, and S. Wright, "Power awareness in network design and routing," in Proceedings of the IEEE INFOCOM-The 27th Conference on Computer Communications, pp. 457-465, Phoenix, AZ, USA, April 2008.

[33] N. C. Nguyen, P. Wang, D. Niyato, Y. Wen, and Z. Han, "Resource management in cloud networking using economic analysis and pricing models: a survey," IEEE Communications Surveys \& Tutorials, vol. 19, no. 2, pp. 954-1001, 2017.

[34] L. Zhang, K. Li, C. Li, and K. Li, "Bi-objective workflow scheduling of the energy consumption and reliability in heterogeneous computing systems," Information Sciences, vol. 379, pp. 241-256, 2017.

[35] Y. Liu, H. S. Seah, and G. Shou, "Constrained energy-efficient routing in time-aware road networks," GeoInformatica, vol. 21, no. 1, pp. 89-117, 2017.

[36] J. Rak, M. Pickavet, K. S. Trivedi et al., "Future research directions in design of reliable communication systems," Telecommunication Systems, vol. 60, no. 4, pp. 423-450, 2015.

[37] Y. Cui, S. Xiao, X. Wang et al., "Performance-aware energy optimization on mobile devices in cellular network," IEEE Transactions on Mobile Computing, vol. 16, no. 4, pp. 10731089, 2017. 
[38] K. Wang, J. Yu, Y. Yu et al., "A survey on energy internet: architecture, approach, and emerging technologies," IEEE Systems Journal, vol. 12, no. 3, pp. 2403-2416, 2017.

[39] H. Bast, D. Delling, A. Goldberg et al., "Route planning in transportation networks," in Algorithm Engineering, pp. 19-80, Springer, Berlin, Germany, 2016.

[40] F. Wu, "Integration of software reliability engineering, risk management and safety assurance case," in Proceedings of the IEEE International Symposium on Software Reliability Engineering Workshops (ISSREW), pp. 169-192, Pasadena, CA, USA, November 2013.

[41] V. Balasubramanian, M. Aloqaily, F. Zaman, and Y. Jararweh, "Exploring computing at the edge: a multi-interface system Architecture enabled mobile device cloud," in Proceedings of the IEEE 7th International Conference on Cloud Networking (CloudNet), pp. 1-4, Tokyo, Japan, October 2018.

[42] A. Sharma and R. Kumar, "Risk-energy aware service level agreement assessment for computing quickest path in computer networks," International Journal of Reliability and Safety, vol. 13, no. 1-2, p. 96, 2019.

[43] H. I. Calvete, L. del-Pozo, and J. A. Iranzo, "Dealing with residual energy when transmitting data in energy-constrained capacitated networks," European Journal of Operational Research, vol. 269, no. 2, pp. 602-620, 2018.

[44] A. Chen, H. Yang, H. K. Lo, and W. H. Tang, "A capacity related reliability for transportation networks," Journal of Advanced Transportation, vol. 33, no. 2, pp. 183-200, 1999.

[45] V. Kounev, M. Lévesque, D. Tipper, and T. Gomes, "Reliable communication networks for smart grid transmission systems," Journal of Network and Systems Management, vol. 24, no. 3, pp. 629-652, 2016.

[46] R. K. Ahuja, T. L. Magnanti, and J. B. Orlin, Network Flows: Theory, Algorithms, and Applications, MIT Sloan School of Management, Cambridge, MA, USA, 1993.

[47] W.-C. Yeh and M. El Khadiri, "A new universal generating function method for solving the single \$(d,|tau) \$-quick-path problem in multistate flow networks," IEEE Transactions on Systems, Man, and Cybernetics-Part A: Systems and Humans, vol. 42, no. 6, pp. 1476-1484, 2012.

[48] A. Sharma and R. Kumar, "Service-level agreement-energy cooperative quickest ambulance routing for critical healthcare services," Arabian Journal for Science and Engineering, vol. 44, no. 4, pp. 3831-3848, 2019.

[49] M. L. Fredman and R. E. Tarjan, "Fibonacci heaps and their uses in improved network optimization algorithms," Journal of the ACM, vol. 34, no. 3, pp. 596-615, 1987. 


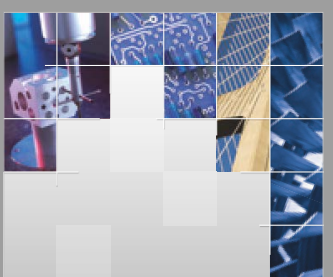

\section{Enfincering}
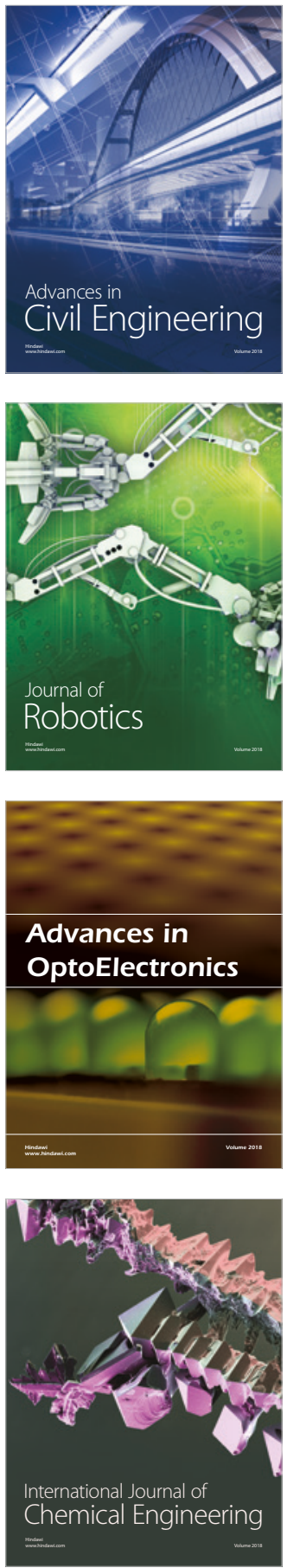

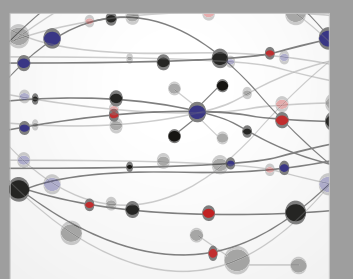

\section{Rotating \\ Machinery}

The Scientific World Journal

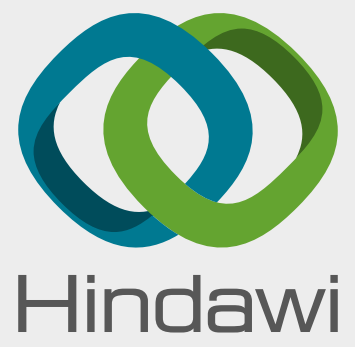

Submit your manuscripts at

www.hindawi.com
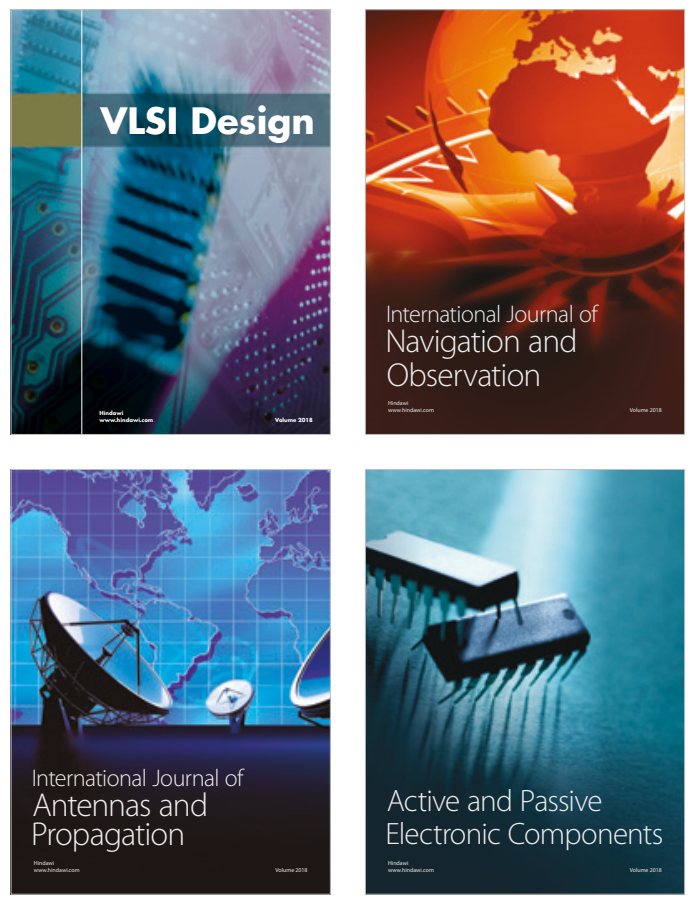
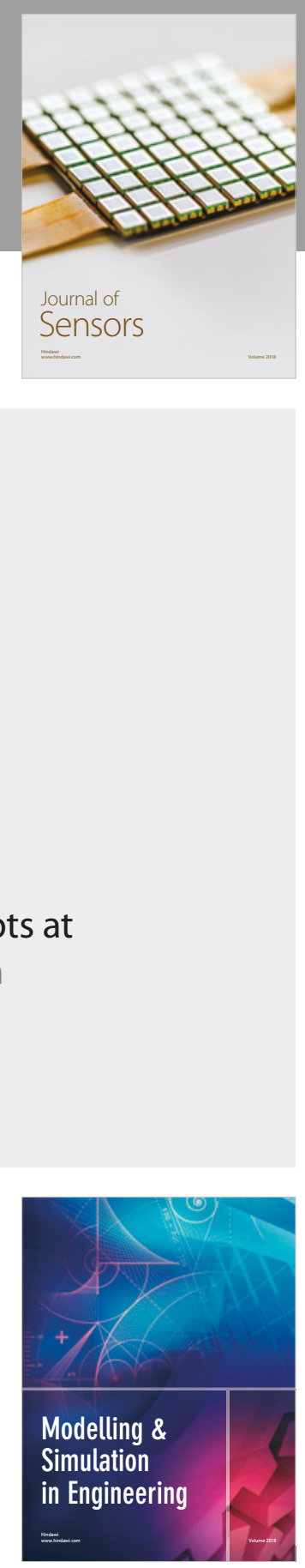

\section{Advances \\ Multimedia}
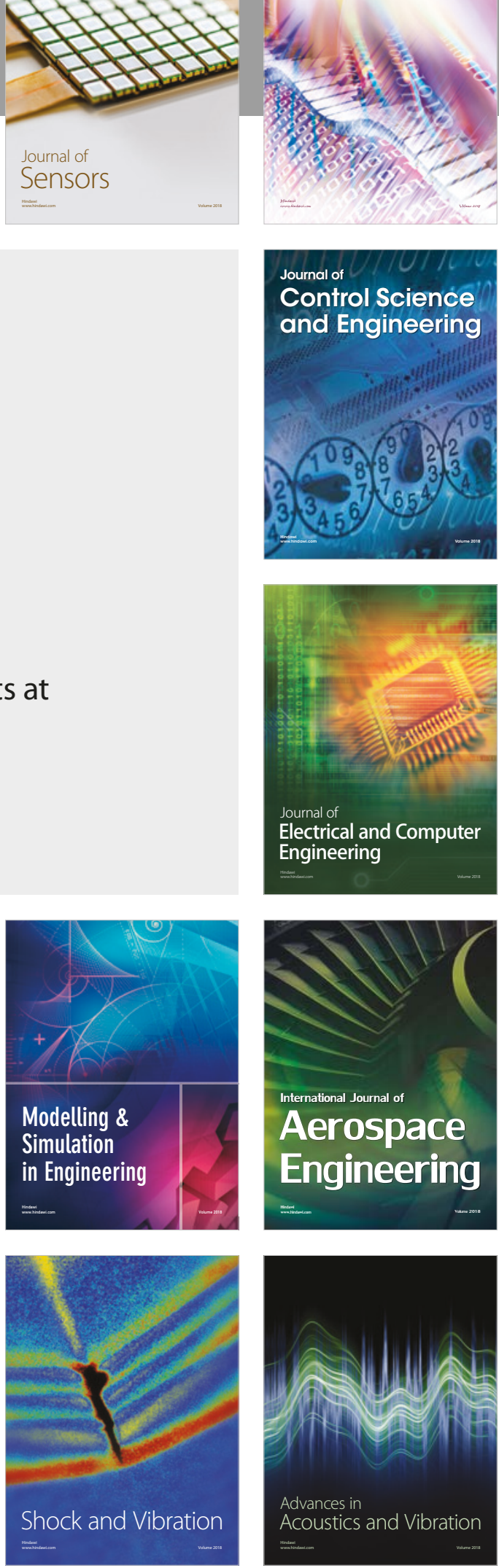